Arq. Bras. Med. Vet. Zootec., v.69, n.5, p.1139-1144, 2017

\title{
Avaliação do fluxo de saída do ventrículo esquerdo em gatos anestesiados submetidos ao pneumoperitônio e à posição deTrendelenburg
}

[Evaluation left ventricle Outflow in cats anesthetized submitted to pneumoperitoneum and Trendelenburg position]

\author{
M.L. Magalhães ${ }^{1}$, L.T. Nishimura ${ }^{1}$, A.T. Souza ${ }^{1}$, C.F. Magalhães ${ }^{2}$, E. Mattos Júnior ${ }^{1}$, \\ C.S. Honsho ${ }^{1}$, D. Paulino Júnior \\ ${ }^{1}$ Universidade de Franca - Unifran - Franca, SP \\ ${ }^{2}$ Instituto Federal de Educação Ciência e Tecnologia do Triângulo Mineiro - Patos de Minas, MG
}

\begin{abstract}
RESUMO
Objetivou-se avaliar os efeitos do pneumoperitônio e da posição de Trendelenburg sobre o fluxo de saída do ventrículo esquerdo em gatos anestesiados. Quatorze gatos foram alocados aleatoriamente em dois grupos, ambos submetidos ao pneumoperitônio com $10 \mathrm{mmHg}$ de dióxido de carbono $\left(\mathrm{CO}_{2}\right)$. No grupo controle $(\mathrm{GC} n=7)$, os animais foram submetidos apenas ao pneumoperitônio e, no grupo Trendelenburg (GTREN $\mathrm{n}=7$ ), os animais foram colocados em cefalodeclive $20^{\circ}$ após o pneumoperitônio. A indução anestésica foi realizada com isoflurano, utilizando-se caixa de indução. Posteriormente, os animais foram mantidos sob anestesia inalatória com o mesmo fármaco. Foram avaliados a velocidade do fluxo de saída do ventrículo esquerdo (VFSVE), os gradientes máximo (GmáxSVE) e médio (GmédSVE) de pressão e a integral velocidade-tempo (IVT). Os parâmetros foram mensurados nos momentos T0 (basal), antes da insuflação; T5 (cinco), T15 (quinze) e T30 (trinta) minutos após a insuflação. Os resultados mostraram um aumento da VFSVE no GC, em T15 e T30 ( $\mathrm{P}=0,024)$, e um aumento do GmáxSVE no GC, em T30 $(\mathrm{P}=0,045)$. As variáveis não se alteraram significativamente em nenhum momento no GTREN. Dessa forma, conclui-se que a posição de Trendelenburg favoreceu o sistema cardiovascular, preservando os índices de fluxo sanguíneo na saída do ventrículo esquerdo.
\end{abstract}

Palavras-chave: gato, $\mathrm{CO}_{2}$, Doppler, fluxo de saída, Trendelenburg

\begin{abstract}
The aim of this study was to evaluate the effects of pneumoperitoneum and Trendelenburg position on the left ventricular outflow in anesthetized cats. Fourteen cats were randomly divided into two groups, both submitted to pneumoperitoneum of $10 \mathrm{mmHg}$ with carbon dioxide (CO2), and in the control group (GC $n$ $=7)$ the animals were subjected only to pneumoperitoneum and the Trendelenburg group $(n=7$ GTREN) the animals were placed in cefalodeclive $20^{\circ}$ after pneumoperitoneum. Anesthesia of the animals was performed with isoflurane using induction box, keeping the animals under inhalation anesthesia with the same drug. We evaluated the speed of the left ventricular outflow (VFSVE), the maximum pressure gradient (GmáxSVE), mean pressure gradient (GmédSVE) and velocity-time integrals (IVT). The parameters were measured in time, T0 (baseline), before the insufflation; T5 (five); T15 (fifteen) and T30 (thirty) minutes after inflation. The results showed an increase in VFSVE in GC, T15 and T30 $(p=0,024)$ and an increase in GmáxSVE in GC in T30 ( $p=0,045)$. The variables did not change significantly at any time in GTREN. Thus, it is concluded that the Trendelenburg position favored the cardiovascular system, preserving blood flow rates in the left ventricular outflow.
\end{abstract}

Keywords: cat, CO2, doppler, outflow, Trendelenburg

Recebido em 13 de outubro de 2016

Aceito em 3 de dezembro de 2016

E-mail: marcosmagalhaes9@gmail.com 


\section{INTRODUÇÃO}

A busca por procedimentos minimamente invasivos, objetivando uma melhora nos diagnósticos e nas terapias cirúrgicas, destaca a videocirurgia como uma das alternativas do médico veterinário para o tratamento de diversas afecções, por apresentar vantagens em relação aos procedimentos cirúrgicos tradicionais. Não obstante as características minimamente invasivas dos procedimentos videoassistidos, complicações trans e pós-operatórias advindas do estabelecimento do pneumoperitônio e do posicionamento de Trendelenburg já foram estudadas em humanos e modelos animais, entretanto pouco se sabe sobre o comportamento hemodinâmico dos gatos submetidos a esse procedimento.

Após o estabelecimento do pneumoperitônio para realização de uma videocirurgia abdominal, há um aumento na pressão intra-abdominal (PIA), o que ocasiona o deslocamento cranial do diafragma (Wahba e Mamazza, 1993; Fagundes et al., 2001). Isso leva ao aumento da pressão intratorácica e, como consequência, reduz a complacência pulmonar, repercutindo diretamente sobre o sistema cardiovascular, pois eleva a resistência vascular sistêmica, reduzindo o retorno venoso e o débito cardíaco (Hirvonen et al., 1995; Ortega et al., 1996). Adicionalmente ao pneumoperitônio, o posicionamento de Trendelenburg, tendo sido utilizado com o fim de facilitar o deslocamento cranial das vísceras e melhorar o campo visual em cirurgias pélvicas, no entanto, essa associação é conhecida, em humanos, por causar hipertensão intracraniana (Halverson et al., 1998).

O coração está apoiado no diafragma e é sustentado pelos grandes vasos torácicos no espaço mediastinal médio e sua base é composta pelos principais vasos de saída do fluxo sanguíneo. Compreender os efeitos compressivos do pneumoperitônio sobre esse órgão e os efeitos de seu deslocamento em direção à sua base, quando em posição de Trendelenburg, é fundamental para verificar alterações transitórias na hemodinâmica e, até mesmo, uma possível obstrução dinâmica na via de saída do ventrículo esquerdo, advinda do cefalodeclive. Por conseguinte, a avaliação ecocardiográfica quantitativa e qualitativa (subjetiva) do fluxo de saída do ventrículo esquerdo é o exame de escolha para fornecer informações precisas sobre os índices de fluxo sanguíneo na saída do ventrículo esquerdo.

Em razão das peculiaridades anatomofisiológicas dos gatos, do seu reduzido peso corporal, quando comparado aos humanos e a outras espécies animais, da escassez de estudos referentes à associação do pneumoperitônio e do posicionamento de Trendelenburg nessa espécie, esta pesquisa objetivou esclarecer se o pneumoperitônio, associado ou não ao posicionamento de Trendelenburg, é capaz de produzir alterações sobre o fluxo de saída do ventrículo esquerdo.

\section{MATERIAL E MÉTODO}

A presente pesquisa foi realizada sob a anuência da Comissão de Ética na Utilização de Animais da Universidade de Franca (Protocolo $n^{\circ}$ 036/13). Foram utilizados 14 gatos, machos $(n=3)$ e fêmeas $(n=11)$, hígidos, sem raça definida, adultos, com peso médio de 2,8 $\pm 0,9 \mathrm{~kg}$.Os animais foram submetidos a estudo randomizado, distribuídos em dois grupos $(n=7)$ designados de GC (grupo controle com animais em decúbito dorsal submetidos ao pneumoperitônio de $10 \mathrm{mmHg}$ com $\mathrm{CO}_{2}$ ) e GTREN (animais em decúbito dorsal submetidos ao pneumoperitônio de $10 \mathrm{mmHg}$ com $\mathrm{CO}_{2}$ associado à posição de Trendelenburg de $20^{\circ}$ ).

Os animais foram induzidos à anestesia com isoflurano (Isoflurano ${ }^{\circledR}$, Instituto BioChimico Indústria Farmacêutica, RJ, Brasil) fração inspirada de 3,5\%, diluído $100 \mathrm{~mL} / \mathrm{kg} / \mathrm{min}$, utilizando-se caixa de indução. Após apresentarem relaxamento mandibular, foram entubados com sonda traqueal conectada ao circuito de anestesia e mantidos com isoflurano fração inspirada de $1,5 \%$ e fluxo de $100 \mathrm{~mL} / \mathrm{kg} / \mathrm{min}$, diluído em $100 \%$ de oxigênio.

Em ambos os grupos, após o preparo do campo operatório, realizou-se incisão cutânea para localização da linha alba na região umbilical; em seguida, foi inserida a agulha de Veress para instituição do pneumoperitônio com $\mathrm{CO}_{2}$ de $10 \mathrm{mmHg}$, com fluxo de $1 \mathrm{~L} / \mathrm{min}$. Os animais do GTREN foram submetidos à posição de Trendelenburg $20^{\circ} \operatorname{logo}$ após a instituição do pneumoperitônio. 
Para a avaliação ecocardiográfica da via de saída do ventrículo esquerdo, utilizou-se o ecocardiógrafo (EnVisor CHD, PHILIPS, Andover MA, USA) com transdutor setorial de alta densidade, multifrequencial $(5,0-8,5 \mathrm{MHz})$, em modo bidimensional. Para a análise do fluxo sanguíneo, utilizou-se o Doppler colorido para avaliação qualitativa, com vista a identificar alterações no padrão e na tonalidade do fluxo sanguíneo, e o Doppler pulsado para a avaliação quantitativa. As variáveis foram aferidas nos momentos T0 (basal), imediatamente antes da insuflação; T5 (cinco), T15 (quinze) e T30 (trinta) minutos após a insuflação.

As imagens ecocardiográficas foram obtidas pela janela paraesternal esquerda, corte apical cinco câmaras. Mensuraram-se as variáveis quantitativas VFSVE (velocidade máxima do fluxo de saída do ventrículo esquerdo), GmáxSVE (gradiente máximo de pressão na saída do ventrículo esquerdo, GmédSVE (gradiente médio de pressão na saída do ventrículo esquerdo) e IVT (integral velocidadetempo). Esses índices foram obtidos e calculados mediante software instalado no aparelho ecocardiográfico, após ser traçado manualmente o contorno do espectro dos fluxos analisados (Fig. 1). As medidas foram feitas a partir de três ciclos cardíacos consecutivos, e o resultado considerado foi a média dos valores obtidos.

A estatística foi obtida por meio do programa computacional GraphPad PRISM v. 5 (GraphPad Software, Inc, La Jolla, CA, USA), cujos dados da avaliação quantitativa foram apresentados em forma de média, com respectivo desvio-padrão. Esses dados foram comparados dentro dos grupos nos diferentes momentos da avaliação pela análise de variância (ANOVA). Após o teste de Bonferroni foi empregado o test-t pareado para análise entre grupos, dentro dos diferentes momentos. Todos os testes foram realizados considerando-se nível de significância de 5\%.

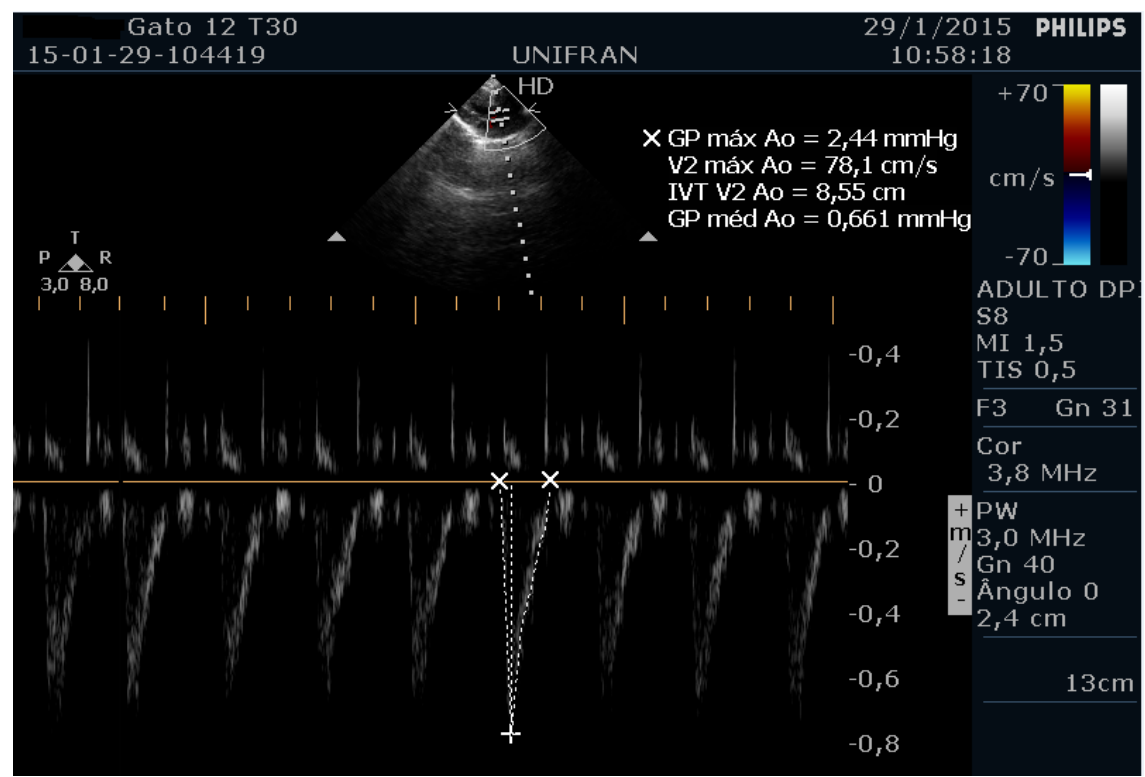

Figura 1. Avaliação, com Doppler pulsado, do fluxo de saída do ventrículo esquerdo de gato anestesiado. Observa-se o contorno manual do espectro para a obtenção da velocidade máxima do fluxo na saída do ventrículo esquerdo (V2 máx Ao), do gradiente máximo de pressão na via de saída do ventrículo esquerdo (GP máx Ao), do gradiente médio de pressão na via de saída do ventrículo esquerdo (GP méd Ao) e da integral velocidade-tempo (IVT V2 Ao).

\section{RESULTADOS E DISCUSSÃO}

A habilidade da ecocardiografia Doppler em estimar a velocidade dos fluxos sanguíneos de forma não invasiva abriu novas áreas de investigação para a detecção e quantificação de várias lesões cardíacas (Petrus et al., 2010). Ela fornece informações qualitativas, em tempo real, sobre a presença e direção do fluxo sanguíneo em um vaso e também informações quantitativas, como a velocidade do fluxo sanguíneo, 
permitindo a análise da impedância e da resistividade vascular (Carvalho et al., 2008b).

Durante o estudo, as imagens ecocardiográficas foram obtidas pela janela paraesternal esquerda e pelo corte apical cinco câmaras. Os animais permaneceram em decúbito dorsal durante todo o procedimento, tendo em vista que o estudo objetivava, naquele momento, avaliar possíveis efeitos adversos do pneumoperitônio e a sua associação com o posicionamento de Trendelenburg no transoperatório. Para tal, utilizou-se o Doppler color, a fim de verificar o padrão e a tonalidade do fluxo de saída do ventrículo esquerdo, já que, na ecocardiografia diagnóstica, como rotina, usa-se o decúbito lateral esquerdo. Esse posicionamento não constituiu um fator limitante para o experimento, visto que todas as variáveis foram mensuradas de forma equânime pelo mesmo observador. Além disso, para situações em que se faz necessária a avaliação do arco aórtico e da aorta descendente, o paciente deve estar em decúbito dorsal (Thomas, 1984; Feigenbaum, 1986). Por outro lado, com o Doppler pulsado é possível caracterizar a fluxometria padrão de cada vaso sanguíneo, além de verificar mudanças no padrão espectral das ondas, obtendo-se significado clínico a possíveis alterações (Szatmári et al., 2001). Portanto, cada vaso apresenta uma assinatura ou um traçado espectral característico, que permite identificá-lo com precisão (Carvalho et al., 2008a).
Optou-se por relacionar o aumento ou a diminuição da IVT a alterações reais no débito cardíaco, tendo como base importantes sustentações de que a IVT está diretamente associada ao volume sistólico (Bonagura e Fuentes, 2004). Assim, aumentos na IVT representam aumento do fluxo ou do volume, enquanto a diminuição na IVT representa diminuição do fluxo ou do volume (Boon, 1998). Além disso, segundo Uehara (1993), imprecisões na medida da área valvar, agravadas pela movimentação do coração durante a respiração, podem comprometer os valores do débito cardíaco.

Os resultados da avaliação quantitativa estão apresentados na Tab. 1. Analisando-se a VFSVE, observou-se aumento nos momentos T15 e T30 no GC $(\mathrm{P}=0,024)$, não ocorrendo diferença estatística dessa variável no GTREN (P>0,05). Seguindo essa tendência, houve aumento do GmáxSVE no momento T30 do CG $(\mathrm{P}=0,045)$. Alterações na fisiologia pulmonar, advindas dos efeitos adversos no estabelecimento do pneumoperitônio, foram documentadas em humanos, entre elas, o aumento na PIA que desloca cranialmente o diafragma, aumentando a pressão intratorácica e reduzindo a complacência pulmonar. Essas alterações repercutem diretamente sobre o sistema cardiovascular, pois elevam a resistência vascular sistêmica, reduzem o retorno venoso e o débito cardíaco, consequentemente (Wahba e Mamazza,1993; Fagundes et al., 2001).

Tabela 1. Médias, desvio-padrão e valores de probabilidade $(\mathrm{P})$ das variáveis ecocardiográficas nos diferentes momentos, em gatos anestesiados com isoflurano

\begin{tabular}{ccccccc}
\hline Variável & Grupos & T0 & T5 & T15 & T30 & P(anova) \\
\hline \multirow{2}{*}{ VFSVE $^{1}(\mathrm{~cm} / \mathrm{s})$} & GC & $64,20 \pm 7,83$ & $72,10 \pm 10,51$ & $77,65 \pm 10,11^{*}$ & $77,67 \pm 12,07 *$ & 0,024 \\
& GTREN & $66,00 \pm 7,17$ & $67,13 \pm 10,47$ & $70,19 \pm 15,39$ & $69,91 \pm 9,49$ & 0,558 \\
& P(teste t) & 0,756 & 0,379 & 0,105 & 0,223 & \\
GmáxSVE$^{2}(\mathrm{mmHg})$ & GC & $1,67 \pm 0,40$ & $2,12 \pm 0,58$ & $2,32 \pm 0,63$ & $2,46 \pm 0,73 *$ & 0,045 \\
& GTREN & $1,76 \pm 0,40$ & $1,84 \pm 0,59$ & $2,06 \pm 0,88$ & $1,99 \pm 0,54$ & 0,456 \\
& P(teste t) & 0,766 & 0,386 & 0,268 & 0,213 & \\
GmédSVE $^{3}(\mathrm{mmHg})$ & GC & $0,61 \pm 0,14$ & $0,81 \pm 0,26$ & $0,81 \pm 0,26$ & $0,80 \pm 0,27$ & 0,156 \\
& GTREN & $0,59 \pm 0,17$ & $0,70 \pm 0,22$ & $0,73 \pm 0,33$ & $0,66 \pm 0,28$ & 0,223 \\
& P(teste t) & 0,820 & 0,435 & 0,328 & 0,297 & \\
IVT $^{4}(\mathrm{~cm})$ & GC & $6,79 \pm 1,42$ & $8,37 \pm 1,50$ & $8,42 \pm 1,46$ & $8,08 \pm 1,96$ & 0,101 \\
& GTREN & $7,05 \pm 1,48$ & $7,15 \pm 1,17$ & $8,19 \pm 1,81$ & $7,66 \pm 2,06$ & 0,177 \\
& P(teste t) & 0,798 & 0,194 & 0,841 & 0,733 & \\
\hline
\end{tabular}

${ }^{1}$ Velocidade máxima do fluxo de saída do ventrículo esquerdo; ${ }^{2}$ gradiente máximo de pressão na saída do ventrículo esquerdo; ${ }^{3}$ gradiente médio de pressão na saída do ventrículo esquerdo; ${ }^{4}$ integral velocidade-tempo. * Diferença estatística significativa entre os diferentes momentos em relação ao T0 (basal). 
Chiu et al. (1995) relataram efeitos da compressão de vasos abdominais causada pela instituição do pneumoperitônio sobre os rins. Esses autores observaram que a redução do fluxo sanguíneo na cortical renal contribui para elevação na produção do hormônio antidiurético, hipersecreção de renina e elevação do nível de aldosterona sérica. Esses achados, por si só, poderiam justificar o aumento da VFSVE e do GmáxSVE observado no GC, pois seriam consequência da mudança imediata nos parâmetros hemodinâmicos e neuro-hormonais ocasionada pela ativação dos mecanismos compensatórios do sistema renina-angiotensinaaldosterona (SRAA), levando ao aumento da resistência vascular periférica e do volume sanguíneo (pré e pós-carga). No entanto, a função cardiovascular também pode sofrer alteração devido à interação primária do $\mathrm{CO} 2$ com as fibras miocárdicas (Rasmussen et al., 1978).

Como não foram observadas diferenças estatísticas na IVT em ambos os grupos $(\mathrm{P}>0,05)$, acredita-se que o aumento da VFSVE e do GmáxSVE no GC decorreu de uma ação primária do sistema cardiovascular para compensar o aumento da resistência vascular sistêmica e da redução no retorno venoso advindo da instituição do pneumoperitônio para, assim, manter a perfusão tecidual, antes, até mesmo, do aumento no volume sistólico, este ocasionado pela elevação da pré e pós-carga, repercussões hemodinâmicas decorrentes da ativação do SRAA. Por conseguinte, o tempo médio do estudo em questão $(39 \pm 9$ min desde a indução) não modificou os valores da IVT. Estudos posteriores, com o tempo de transoperatório superior ao proposto por este experimento, poderão elucidar se, além de T30, ocorreria um aumento significativo na IVT, caracterizando aumento do volume sistólico, fruto de uma importante repercussão hemodinâmica.

Não se observaram diferenças estatísticas em nenhuma das variáveis no GTREN $(\mathrm{P}>0,05)$ (Tab. 1). Esses achados corroboram os estudos de Miller (1940) e Johnson e Henderson (2004), que afirmam que posturas em cefalodeclive favorecem a circulação, melhoram o retorno venoso, mantêm o débito cardíaco e a perfusão dos órgãos vitais. Logo, a posição de Trendelenburg leva a uma melhor acomodação dos órgãos, contribuindo para a descompressão abdominal, o que proporciona efeitos benéficos ao sistema circulatório e à pressão arterial sistêmica (Caldwell e Ricotta, 1987).

\section{CONCLUSÃO}

Os resultados desta pesquisa mostraram que a instituição do pneumoperitônio isoladamente aumentou a VFSVE e o GmáxSVE, mas sua associação à posição de Trendelenburg favoreceu o sistema cardiovascular, preservando os índices de fluxo sanguíneo na saída do ventrículo esquerdo, além de não ter causado nenhum tipo de obstrução dinâmica na via de saída do ventrículo esquerdo.

\section{AGRADECIMENTO}

Os autores agradecem à Fundação de Amparo à Pesquisa do Estado de São Paulo.

\section{REFERÊNCIAS}

BONAGURA, J.D.; FUENTES, V.L. Ecocardiografia. In: ETTINGER, S.J.; FELDMAN, E.C. (Ed.). Tratado de medicina interna veterinária: doenças do cão. 5.ed. São Paulo: Guanabara Koogan, 2004. p.884-925.

BOON, J.A. Evaluation of size, function, and hemodynamics. In: BOON, J.A. (Ed.). Manual of veterinary echocardiography. Baltimore: Williams and Wilkins, 1998. p.151-260.

CALDWELL, C.B.; RICOTTA, J.J. Changes in visceral blood flow with elevated intraabdominal pressure. J. Surg. Res., v.43, p.14-20, 1987.

CARVALHO, C.F.; CHAMMAS, M.C.; CERRI, G.G. Princípios físicos do Doppler em ultrasonografia. Ciênc. Rural., v.38, p.872-879, 2008a.

CARVALHO, C.F.; CHAMMAS, M.C.; STERMANN, F.A. et al. Ultra-sonografia dúplex-Doppler na avaliação morfológica e hemodinâmica das artérias aorta e mesentérica cranial em cães. Braz. J. Vet. Res. Anim. Sci., v.45, p.24-31, 2008 b. 
CHIU, A.W.; CHANG, L.S.; BIRKETT, D.H.; BABAYAN, R.K. The impact of pneumoperitoneum, pneumoretroperitoneum, and gasless laparoscopy on the systemic and renal hemodynamics. J. Am. Coll. Surg., v.181, p.397-406, 1995.

FAGUNDES, M.A.V.; MONTERO, E.F.S.; NOVO, N.F.; BANDEIRA, O.P. Efeitos do pneumoperitônio em ratos. Acta Sci. Health Sci., v.23, p.703-707, 2001.

FEIGENBAUM, H. The echocardiographic examination. In: FEIGENBAUM, H. (Ed.). Echocardiography. 4.ed. Philadelphia: Lea \& Febiger, 1986. p.50-126.

HALVERSON, A.; BUCHANAN, R.; JACOBS, L. et al. Evaluation of mechanism of increased intracranial pressure with insufflation. Surg. Endosc., v.12, p.266-269, 1998.

HIRVONEN, E.A.; NUUTINEN, L.S.; KAUKO, M. Ventilatory effects blood gas changes and oxygen consumption during laparoscopic hysterectomy. Anesth. Analg., v.80, p.961-966, 1995.

JOHNSON, S.; HENDERSON, S.O. Myth: The trendelenburg position improves circulation in cases of shock. Can. J. Emer. Med., v.6, p.48-49, 2004.

MILLER, A.H. Surgical posture with symbols for its record on the anesthetist's chart. Anesthesiology, v.1, p.241-245, 1940.
ORTEGA, A.; PETERS, J.; INCARBONE, R.; ESTRADA, L. et al. A prospective randomized comparison of the metabolic and stress hormonal responses of laparoscopic and open cholecystectomy. J. Am. Coll Surg., v.183, p.249-256, 1996.

PETRUS, L.C.; OLIVEIRA, V.M.C.; PEREIRA, G.G.; LARSSON, M.H.M.A. Avaliação dos fluxos das valvas aórtica e pulmonar com ecocardiografia Doppler pulsátil em cães clinicamente sadios. Pesqui. Vet. Bras., v.30, p.586-592, 2010.

RASMUSSEN, J.P.; DAUCHOT, P.J.; DePALMA, R.G. et al. Cardiac function and hypercarbia. Arch. Surg., v.113, p.1196-1200, 1978.

SZATMÁRI, V. SÓTONYI, P.; VÖRÖS, K. Normal duplex Doppler wave forms of major abdominal blood vessels in dogs: a review. Vet. Radiol. Ultrasound, v.42, p.93-107, 2001.

WAHBA, R.W.; MAMAZZA, J. Ventilatory requirements during laparoscopic cholecystectomy. Can. J. Anesth., v.40, p.206210, 1993.

THOMAS, W.P. Two-dimensional, real-time echocardiography in the dog. Vet. Radiol. Ultrasound, v.25, p.50-64, 1984.

UEHARA, Y. An attempt to estimate the pulmonary artery pressure in dogs by means of pulsed Doppler echocardiography. J. Vet. Med. Sci., v.55, p.307-312, 1993. 